\title{
Can the Forward-Step-Down Test Be Used Reliably in the Clinical Setting to Assess Movement Changes Resulting from Maximal Exertion? A Pilot Study
}

\author{
Erin McCallister \\ Louisiana State University Health Sciences Center - Shreveport, emadar@lsuhsc.edu \\ Daniel W. Flowers \\ Louisiana State University Health Sciences Center - Shreveport, dflowe@Isuhsc.edu
}

Follow this and additional works at: https://nsuworks.nova.edu/ijahsp

Part of the Physical Therapy Commons

\begin{abstract}
Recommended Citation
McCallister E, Flowers DW. Can the Forward-Step-Down Test Be Used Reliably in the Clinical Setting to Assess Movement Changes Resulting from Maximal Exertion? A Pilot Study. The Internet Journal of Allied Health Sciences and Practice. 2020 Jan 01;18(4), Article 7.
\end{abstract}

This Manuscript is brought to you for free and open access by the College of Health Care Sciences at NSUWorks. It has been accepted for inclusion in Internet Journal of Allied Health Sciences and Practice by an authorized editor of NSUWorks. For more information, please contact nsuworks@nova.edu. 


\title{
Can the Forward-Step-Down Test Be Used Reliably in the Clinical Setting to Assess Movement Changes Resulting from Maximal Exertion? A Pilot Study
}

\begin{abstract}
Introduction: Visual assessment of lower extremity mechanics is used frequently in clinical practice, and objective scoring of the visual assessment is beneficial to improve objectivity of patient evaluation. In addition, lower extremity mechanics change with fatigue and these changes may increase the risk of lower extremity injury. The Forward-Step-Down Test (FSDT) is one such objective tool, but its ability to detect changes in movement quality in response to exertion are not known. Methods: This study utilized a repeated-measures design, where the participants were scored on the FSDT before performing the Bruce protocol for an exertion stimulus. The participants were re-scored on the FSDT at one, five, and ten minutes after completing the Bruce protocol. Results: Wilcoxon signed-rank tests showed a significant change in FSDT score between baseline and five minutes post-exertion $(\mathrm{a}<\mathrm{.017})$. Friedman's ANOVA was non-significant across all four assessments. In addition, despite testing healthy young adults, $50 \%$ of participants scored as "poor" movement quality on the initial test. This number increased to $75 \%$ at significant five-minute post-exertion mark. Discussion: Median scores on the FSDT were significantly different at five minutes post-exertion. However, this statistically significant change is of questionable clinical relevance because the median score changed by 0.5 . This small change from 3.5 to 4.0 may not represent a change in overall movement quality from "moderate" to "poor." Results do indicate that the participants in this study overall had poorer than expected movement quality throughout the testing. These results suggest larger data collection and analysis may be warranted for this population and the general population prior to partaking in exercise. Conclusion: The FSDT detects changes in lower extremity mechanics five minutes following a single exertion stimulus. Participants' scores returned to baseline by ten minutes post-exertion. The FSDT may be a viable tool to assess changes in lower extremity movement quality following a single bout of exertion, and may help determine when participants have recovered back to baseline movement quality.
\end{abstract}

\section{Author Bio(s)}

Erin McCallister, PT, DPT, COMT is an Instructor of Physical Therapy at the LSUHSC-Shreveport School of Allied Health Professions in Shreveport, LA. She is a licensed physical therapist and Board-Certified Clinical Specialist in Orthopaedic Physical Therapy.

Daniel Flowers, PT, DPT, PhD is an Assistant Professor of Physical Therapy at the LSUHSC-Shreveport School of Allied Health Professions in Shreveport, LA. He is a licensed physical therapist and BoardCertified Clinical Specialist in Orthopaedic Physical Therapy, and is the Orthopedic Residency Director at LSUHSC-Shreveport.

\section{Acknowledgements}

No acknowledgements. 


\title{
TIAHSP \\ The Internet Joutnal of Allied Health Sciences and Practice
}

Dedicated to allied health professional practice and education

Vol. 18 No. 4 ISSN 1540-580X

\section{Can the Forward-Step-Down Test Be Used Reliably in the Clinical Setting to Assess Movement Changes Resulting from Maximal Exertion? A Pilot Study}

\author{
Erin McCallister \\ Daniel W. Flowers \\ Louisiana State University \\ United States
}

\begin{abstract}
Introduction: Visual assessment of lower extremity mechanics is used frequently in clinical practice, and objective scoring of the visual assessment is beneficial to improve objectivity of patient evaluation. In addition, lower extremity mechanics change with fatigue and these changes may increase the risk of lower extremity injury. The Forward-Step-Down Test (FSDT) is one such objective tool, but its ability to detect changes in movement quality in response to exertion are not known. Methods: This study utilized a repeated-measures design, where the participants were scored on the FSDT before performing the Bruce protocol for an exertion stimulus. The participants were re-scored on the FSDT at one, five, and ten minutes after completing the Bruce protocol. Results: Wilcoxon signed-rank tests showed a significant change in FSDT score between baseline and five minutes post-exertion $(\alpha<.017)$. Friedman's ANOVA was non-significant across all four assessments. In addition, despite testing healthy young adults, $50 \%$ of participants scored as "poor" movement quality on the initial test. This number increased to $75 \%$ at significant five-minute post-exertion mark. Discussion: Median scores on the FSDT were significantly different at five minutes post-exertion. However, this statistically significant change is of questionable clinical relevance because the median score changed by 0.5 . This small change from 3.5 to 4.0 may not represent a change in overall movement quality from "moderate" to "poor." Results do indicate that the participants in this study overall had poorer than expected movement quality throughout the testing. These results suggest larger data collection and analysis may be warranted for this population and the general population prior to partaking in exercise. Conclusion: The FSDT detects changes in lower extremity mechanics five minutes following a single exertion stimulus. Participants' scores returned to baseline by ten minutes post-exertion. The FSDT may be a viable tool to assess changes in lower extremity movement quality following a single bout of exertion, and may help determine when participants have recovered back to baseline movement quality.
\end{abstract}

Keywords: injury, fatigue, lower extremity, biomechanics

(C) The Internet Journal of Allied Health Sciences and Practice, 2020 


\section{INTRODUCTION}

Visual assessment of lower extremity mechanics during a variety of stepping or landing activities is used clinically to observe lower extremity alignment and movement patterns. The forward-step-down test (FSDT) is one visual assessment tool that allows the rater to objectively measure compensations during a step-down task by assigning the participant a score that correlates to good, moderate, or poor movement quality. The scoring criteria and quality categories are described by Park et al. ${ }^{1}$ Unlike other visual assessment techniques that use force platforms or video analysis, neither special equipment nor professionally trained personnel are necessary to assess the criteria outlined in the FSDT. Given the small number of people with access to specialized equipment or professional training, an assessment that does not require special equipment or extensive professional training would be preferable for clinicians working in a typical clinical environment, or those who participate in sport for recreation.

Numerous studies have examined the impact of fatigue or exertion on the performance of sport-related movements. . $^{2-5}$ The tests used in these studies allow the investigator to examine quality of movement and the impact of fatigue on movement patterns; however, the tests may ask participants to perform these tasks while fatigued, potentially placing the participant at risk for injury during the assessment. The FSDT involves a challenging but controlled motion without using cutting, jumping, or landing. Therefore, it may be a safe alternative to assessments that require the participant to perform plyometric drop jumps, cutting, jumping and running, and landing. ${ }^{2-5}$

In addition, poor scores on the FSDT have been shown to correlate with hip abductor weakness and muscle length deficits. ${ }^{1}$ Compared to a bilateral assessment, such as a drop landing, unilateral assessments may better challenge hip stability in the frontal and transverse planes. ${ }^{6}$ Existing literature discusses the impact of weak or fatigued hip abductors on knee position and the increased risk for knee injury. 3,4,7 Therefore, the FSDT effectively assesses impairments that may impact lower extremity injury risk without asking the participant to perform the potentially compromising motions that could lead to injury.

Studies of the FSDT have shown moderate to high intra-rater reliability and high interrater reliability. $1,8,9$ There are no current studies examining the FSDT as a tool to assess changes in movement quality due to fatigue of any cause. Therefore, in this study, the FSDT is used to measure potential changes in movement quality following a single bout of cardiovascular fatigue brought on by a Bruce treadmill protocol.

The aim of this investigation was to determine whether a volitional maximal exertion test, the Bruce treadmill protocol, has an effect on the FSDT, and to investigate how performance on the FSDT changes compared to baseline over multiple time points during recovery from the single fatigue stimulus. Our alternate hypothesis was that participants would demonstrate a change in the score on the FSDT after performance of the Bruce protocol of at least one of the post-exertional assessments, with the null hypothesis being the absence of a significant change.

\section{METHODS}

\section{Study Design}

The study utilized a repeated-measures design, with all participants undergoing both pre- and post-exertion FSDT assessments. The independent variable was the participant's exercised state, with the repeated-measures design selected to help eliminate within-subject factors. The dependent variable was the score on the FSDT pre-and post-exertion. Two physical therapists performed the FSDT assessments. The scorers reviewed scoring guidelines given in the Park article, and practiced scoring the test prior to data collection to improve interrater reliability. ${ }^{1}$

A literature search did not reveal any prior work examining the effects of an activity/intervention on a within-subjects change in FSDT performance. As a result, no within-subjects effects sizes were available for an a priori power-analysis to calculate the necessary sample size. In previous work examining the interrater reliability of the FSDT, Park et al used an effect size of 1.26 based upon the work of Rabin and Kozol. ${ }^{1}$ Rabin and Kozol's work examined the relationship between performance on the FSDT and ankle dorsiflexion range of motion. ${ }^{10}$ The authors determined that using a conservative effect size of 0.5 to complete the a priori power-analysis was appropriate for this study rather than the 1.26 effect size due to the differences in study design between this study and both the Parks et al and Rabin and Kozol studies. The power analysis was performed using G*Power (HeinrichHeine-University Düsseldorf, Düsseldorf, Germany) and used an alpha of .05 and power of 0.80 for a two-tailed hypothesis via a repeated-measures, within factors analysis of variance (ANOVA). This power analysis determined the need for nine participants.

\section{Participants}

The study was approved by the institution's Institutional Review Board and was registered at ClinicalTrials.gov (ID number NCT03495752). The study was conducted in accordance with the Helsinki Declaration. Participants were recruited from the local university via classroom announcements to the physical therapy students. All participants were fully informed on the experimental 
procedure. Following the written informed consent process, each participant completed the American College of Sports Medicine (ACSM) health screening to determine if there was more than a "low risk" of adverse effects if the participant were to partake in vigorous exercise. ${ }^{11}$ The researchers felt this was an acceptable inclusion criterion due to the vigorous nature of a maximal exertion test. The consenting and screening process resulted in seven male and 11 female participants, ages 22-26, cleared to participate in the study. The last inclusion criterion could only be met during the study: to ensure maximal effort during the testing, participants were required to reach within ten beats per minute (bpm) of the participant's age-predicted maximum heart rate. If the participant did not meet this criterion, they were withdrawn from the study. One participant was withdrawn for not meeting the heart rate requirement during the protocol. One participant withdrew before performing the baseline FSDT. Figure 1 illustrates the recruitment process. A total of 16 ( $\mathrm{N}=16,10$ females, 6 males) participants fulfilled all inclusion criteria and completed all data collection.

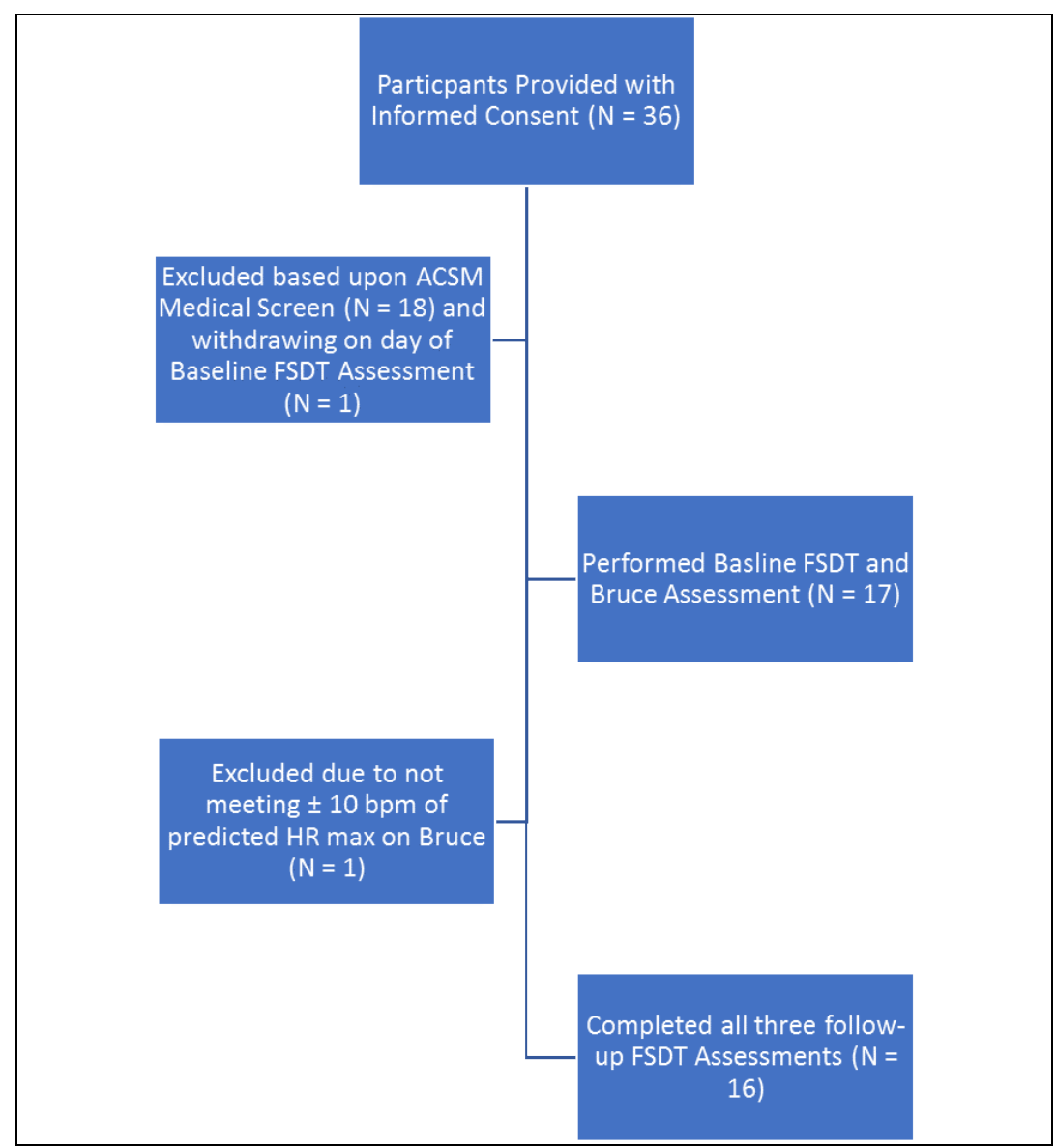

Figure 1. Flowchart of Participant Recruitment

\section{Procedures and Instrumentation}

Following informed consent, age-predicted maximal heart rate was as calculated using the Tanaka formula: maximum heart rate $=208-\left[0.7^{*}\right.$ age $] .{ }^{12}$ The Tanaka formula provides a more accurate prediction of maximum heart rate for this population because it was developed specifically for young, healthy adults. ${ }^{12}$ Prior to starting the maximal exertion protocol, each participant was informed of their predicted maximum heart rate and the minimum heart rate needed to meet the inclusion criterion.

On the testing day, each consented participant's dominant leg was determined. ${ }^{1}$ One investigator read standardized instructions on how to perform the FSDT (see Appendix 1). Participants were not informed on how the test was scored but were given three minutes to practice the test to familiarize themselves with the motion. The initial pre-exertion FSDT was then scored and recorded using the participant's randomized ID number. The FSDT consists of five repetitions of a forward step-down from a $20 \mathrm{~cm}$ step, with one score given for the whole set of five repetitions. Participants are evaluated on their use of arm strategy, trunk lean, pelvic obliquity, front plane knee position, and balance. Table 1 shows the point values assigned to each compensation. ${ }^{1}$ The FSDT was repeated and scored at one, five, and ten minutes after completing the Bruce protocol, which will be called "post-exertion" assessments. 
Table 1. Scoring for the Forward Step Down Test (FSDT).

\begin{tabular}{|l|l|}
\hline FSDT Criteria & Points Given \\
\hline Arm Strategy & $1=$ remove hands from hips for balance \\
\hline Trunk Movement & $1=$ lean for balance \\
\hline Pelvic Plane & $1=$ transverse or frontal plane rotation \\
\hline Knee Position & $\begin{array}{l}1=\text { tibial tuberosity crosses } 2 \text { nd toe } \\
2=\text { crossed medial foot }\end{array}$ \\
\hline Steady Unilateral Stance & $\begin{array}{l}1=\text { non-tested limb touches ground } \\
\text { OR } 1=\text { moves tested foot }\end{array}$ \\
\hline
\end{tabular}

The total score can be categorized to describe the participant's movement quality as good (0-1 point), moderate (2-3 points), or poor ( $\geq 4$ points). Please see Figure $2 a$ and $2 b$ for an example of scoring the FSDT for moderate and poor movement quality. In this study, each investigator recorded the points value for the test, not movement quality designation. To improve accuracy and consistency during scoring frontal knee plane position, both this study and the Park study used a piece of tape to mark the $2^{\text {nd }}$ toe position on the step as well as bare feet to better visualize the landmarks needed for scoring.

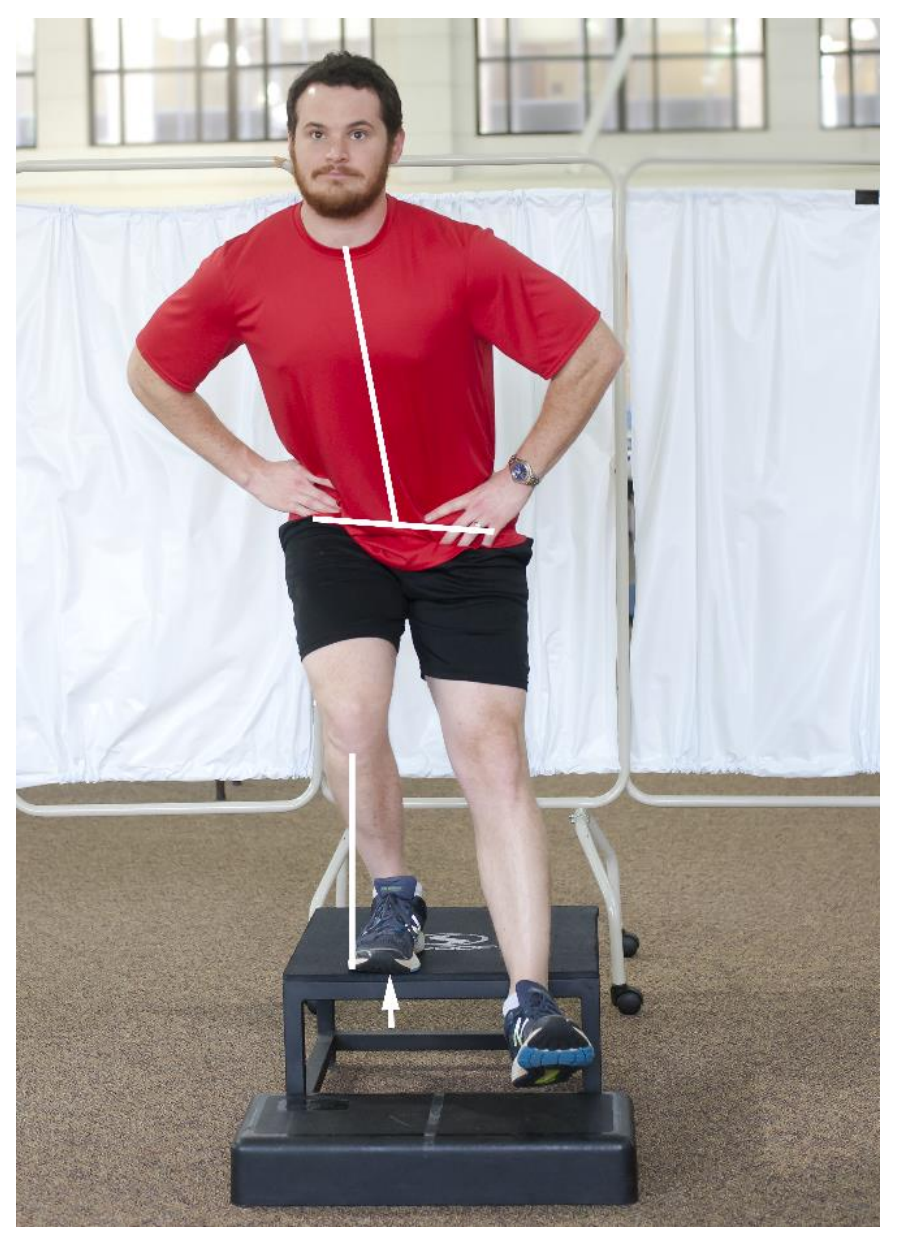

Figure 2a. Scoring of "moderate" quality step down test due to 1 point for trunk movement and 1 point for pelvic plane rotation. 


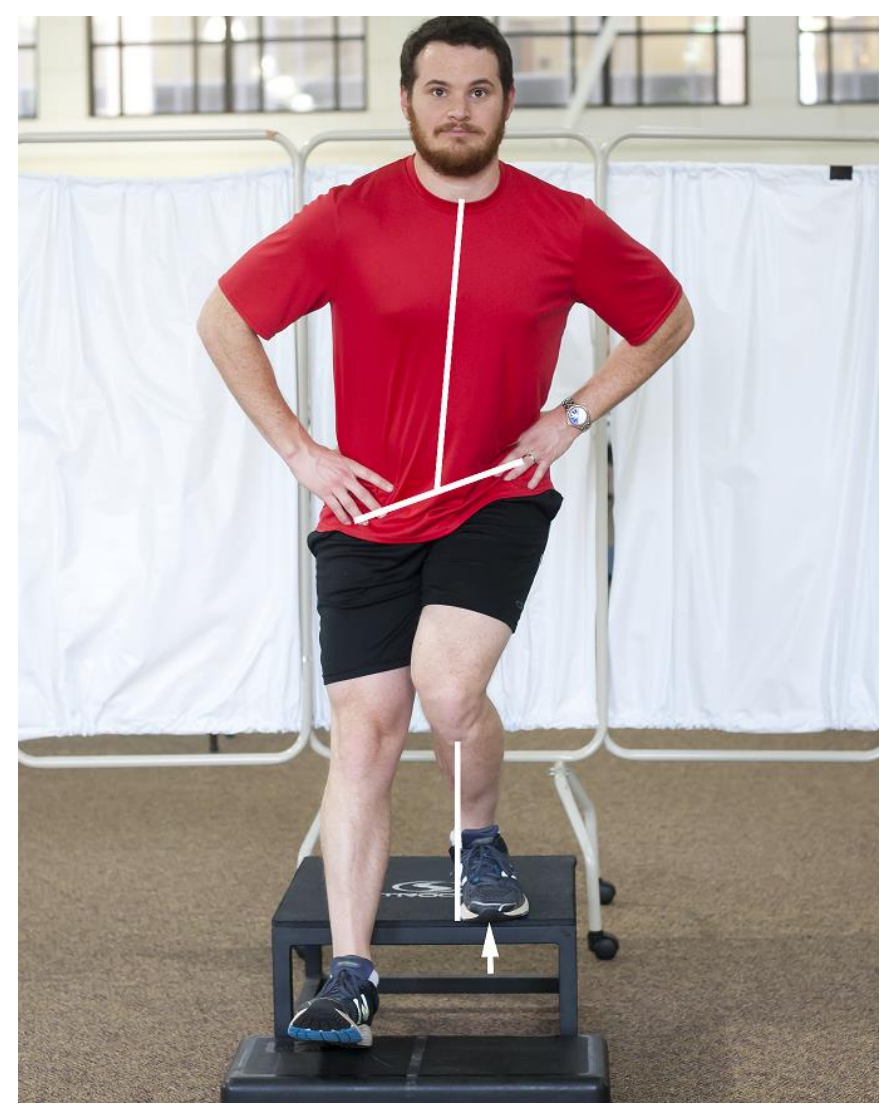

Figure $2 \mathrm{~b}$. Scoring of a "poor" quality step down test due to 1 point for trunk movement, 1 point for pelvic plane rotation, and 2 points for the tibial tuberosity crossing the medial foot.

Each participant performed the FSDT four times throughout the study. Participants performed the baseline FSDT and were reminded of the heart rate needed to meet the last inclusion criterion, then began the maximal exertion Bruce protocol. The Bruce protocol was performed on an HP Cosmos Quasar (HP Cosmos, Germany) pre-programmed with the Bruce protocol. ${ }^{13}$ The investigators monitored heart rate to ensure participant safety and to ensure the last inclusion criterion was met. Heart rate was monitored using Polar heart rate monitors (Polar Electro Inc, Bethpage, NY). The participants' Borg Rating of Perceived Exertion (RPE) was also monitored during the Bruce protocol per convention. The participants were aware that the FSDT would be repeated following the Bruce protocol, so the investigators used heart rate instead of RPE as a metric for maximal exertion to avoid a possible threat to validity of the experimental protocol.

Each participant was in control of how long to continue the Bruce protocol. When the participant reached maximum volitional exertion, the treadmill was stopped and the participant sat for one minute. At one minute, the participant performed the FSDT again. The FSDT was then repeated at five and ten minutes after completion of the Bruce protocol. All four FSDT assessments were scored independently by each investigator and an average score was calculated for each assessment.

\section{Statistical Analysis}

All statistical analyses were conducted using SPSS version 25 (IBM Corporation, Armonk, NY, USA). Due to the ordinal nature of the data, a non-parametric analysis was planned. A Freidman's ANOVA was selected to test for a main effect across all trials due to the repeated-measures nature of the design. Wilcoxon Signed-Rank tests were planned to assess for change in FSDT score compared to baseline as follow-up analyses between baseline and each post-fatigue trial. If significant effects were found, effect sizes were calculated using correlation coefficient and evaluated by Cohen's criteria. Since two evaluators scored each participant, an intraclass correlation coefficient was also calculated to evaluate interrater reliability.

Epidemiological data was also assessed. A percent of participants within each nominal movement category was calculated at baseline and for each post-exertion measurement. 


\section{RESULTS}

A total of 16 participants (6 males, 10 females) completed data collection. A Friedman's ANOVA reported that the score on the FSDT did not significantly change across time when examining the main effect, $X^{2}(3)=5.53, p=.069$. Therefore, multiple Wilcoxon Signed-Rank tests were performed to compare each post-exertion assessment to baseline, for a total of three comparisons. A Bonferroni correction was performed, resulting in a corrected alpha level of .017. There was no significant difference noted between the one-minute ( $M d n=4, T=52.00, p=.043$ ), and 10-minute ( $M d n=3.5, T=38.50, p=.30)$ postassessments when compared to pre-exertion, $(\mathrm{Mdn}=3.5)$. However, there was a significant difference between the pre-exertion and post-exertion assessment performed at five minutes, $(\mathrm{Mdn}=4), T=56.00, p=.015, r=.27$. This difference represents a medium-sized effect. See Figure 3 for a representation of these findings.

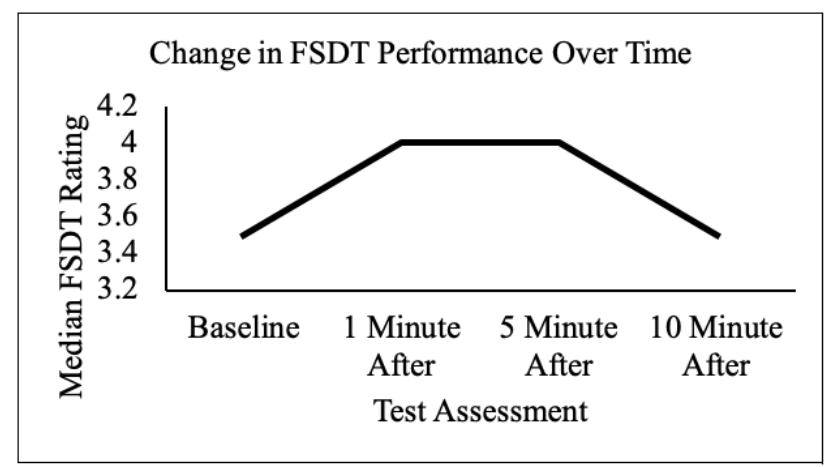

Figure 3. Change in median FSDT scores across time

When examining the data according to the nominal grading nomenclature, none of the participants scored within the "good" movement category, either at baseline or post-exertion. At baseline, half of the participants were considered to have "moderate" quality, while the others had "poor." There was a $50 \%$ increase in the number of participants with "poor" movement quality at the significant five-minute post-exertion test. See Figures 4 and 5.

\section{Pre-Exertion Movement Quality}

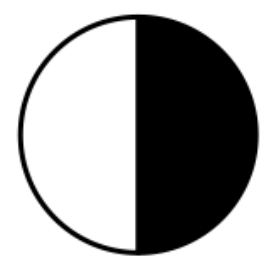

\section{Moderate $\square$ Poor}

Figure 4. Division of participants within movement quality categories pre-exertion.

\section{Post-Exertion Movement Quality}

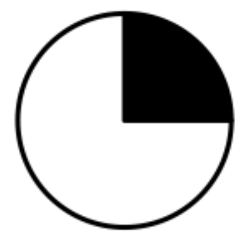

口 Moderate $\square$ Poor

Figure 5. Division of participants within movement quality categories at 5 minutes post-exertion. 
The ratings of both investigators were compared across all four time periods in order to determine the inter-rater reliability (IRR) of the FSDT ratings across the investigation. The intraclass correlation coefficient (ICC) focusing on absolute agreement of the investigators' ratings was used. When interpreting the ICC according to average measures, the ICC was .81, Cl [.68, .88], which indicates high IRR for the ratings of the investigators in this investigation.

\section{DISCUSSION \\ Influence of Maximal Exertion on Test Performance}

The authors hypothesized that one bout of maximal exertion would impact performance on the FSDT at some time point postexertion. The FSDT has been shown to correlate with lower extremity strength. ${ }^{1}$ The selected exertional test, the Bruce protocol, is a treadmill running test that involves cardiovascular as well as muscular exertion because the test uses large increases in ramp and speed to increase the participant's effort. The authors hypothesized that this exertion would result in worse performance on the FSDT when post-exertional scores are compared to pre-exertional baseline scores. However, the effect of the exertion should decrease over time and the participant's performance was expected to return to baseline at a second time point within the testing procedure. Therefore, the overall Friedman's ANOVA would not be significant, as found in this study, if the participants returned to baseline by at least the ten-minute post-exertional FSDT. However, the Wilcoxon Signed-Rank test was also performed to analyze the data for significant change in median score between each post-exertional score and the baseline score. The Wilcoxon tests confirmed the results of the Friedman's ANOVA that there was no significant change between baseline and ten-minute postexertion scores. However, there was a statistically significant change between the pre-and five-minutes post-exertion scores, indicating that participants performed the FSDT worse at five minutes post-exertion compared to their baseline scores.

At the five-minute post-exertion assessment, the Wilcoxon test detected a significant change from baseline score. Therefore, at five minutes after a single bout of muscular and cardiovascular exertion, the FSDT was able to detect a degradation of movement quality in this group of participants. The finding is significant because it has the potential to be clinically meaningful for several groups of people. First, the population tested was a healthy population. Therefore, athletic trainers and coaches may consider using the FSDT as a screening measure to detect a change in baseline test performance to allow a player to return to practice or game play following a rest period. Second, athletic trainers and physical therapists (PT) looking to return an athlete to play may also benefit from using the FSDT. In order to transition athletes back to play, the AT or PT should put a player through training to replicate the stresses experienced during a game or practice. The FSDT may be used to evaluate the player's ability to recover after an exertional stimulus; if the player is not able to return the FSDT score to baseline after more than five minutes of rest, then the player may not be fit to return to the training session.

However, the significance of the post-exertion finding must be critiqued closely. While there is a statistically significant change from baseline score, the median score increased from 3.5 at pre-exertion to 4.0 at five minutes post-exertion. A review of the FSDT scoring criteria shows that a score of 2-3 points indicates "moderate" movement quality, while a score of 4 or greater indicates "poor" movement quality. There is no clear indication of where a score of 3.5 , obtained by mathematically calculating the median score, fits in to the nominal categories of movement quality. Therefore, a change from 3.5 to 4.0 , while statistically significant, may not hold clinical relevance since it may not capture a change in movement quality category.

Other studies have found similar delayed effects of fatigue on performance. A study by Ros et al demonstrated that a one-leg hop test responded to fatigue 15-30 minutes after an aerobic fatigue, but did not respond immediately with a decreased score on the test immediately after the fatigue stimulus. ${ }^{14}$ Poorer scores on a graded jump-landing tasks and dynamic postural control tests were shown after a mixed aerobic-muscular fatigue protocol, but multiple post-test time points were not evaluated. 5,15 However, Steinburg et al found that a postural balance test did respond immediately to an aerobic fatigue test; and recovery did occur by ten minutes post-stimulus, similar to the results of this study. ${ }^{16}$ Therefore, the results of this study are consistent with other results in the literature. However, the effects of fatigue appear to vary based on the task, affected joint, and fatiguing stimulus. Therefore, a battery of tests is most likely to detect and monitor fatigue and recovery for athletes as well as non-athletes participating in exercise or intensive physical therapy.

\section{Epidemiological Data}

An unintended consequence of the study was examining the movement quality of a cohort of physical therapy students. Surprisingly, no student scored in the "good" movement category at their baseline measure. The result is surprising because the initial health screening required the participants to have been performing moderate exercise for at least 30 minutes per day, three days per week for at least three months prior to testing. A score of "moderate" or "poor" on the baseline FSDT indicates area of weakness in either hip or lower extremity musculature or significant muscle flexibility deficits. ${ }^{1}$ Either of these impairments may influence knee valgus during dynamic tasks. ${ }^{1,17-20}$ Therefore, the participants who are performing moderate exercise throughout 
the week may be doing so with non-optimal lower extremity biomechanics, and may be at an increased injury risk if they continue to participate in their selected exercise.

In addition, other studies have shown that students tend to be healthier and more active than their counterparts in the non-academic public. ${ }^{21-23}$ The findings of this study are concerning because half of the participants in this study have baseline poor movement quality and are considered healthy or normal on the preintervention screening measures. The participants are moving with nonoptimal movement patterns despite being more active and healthier than the general public. This is concerning for two reasons. First, it means that the selected participants are performing moderate to vigorous exercise with moderate and poor movement patterns. As stated previously, these movement patterns can alter kinematics at the knee joint and may place the individuals at risk for lower extremity pain or injury either in recreational activity or during their career as physical therapists. Second, if the participants in the study have poor movement patterns and are typically stronger and more fit than their non-academic counterparts, then the general population may have similar or worse overall movement quality on the FSDT. This highlights the importance of getting a physical exam or screening before engaging in physical activity and suggests a possible need for pre-activity interventions to improve muscle strength, muscle length, and motor control.

The current study examined data from a single cohort of Doctor of Physical Therapy students; it may benefit future studies to evaluate larger cohorts of students to obtain more thorough population data. The FSDT is much quicker to perform than detailed strength tests and gives investigators information on a functional movement pattern. Especially for health care professionals, it is important for injury prevention to have optimal strength for lifting patients, performing transfers, or other on-the-job physical activity. A larger pool of data would allow investigators to assess if a general fitness program would benefit the general physical therapy student population, and could provide justification for the cost of such a program.

Lastly, this study could also be expanded to examine the movement quality and response to exertion in the general population. Given that previous studies have found students are more active than the general population, the finding of overall moderate to poor movement quality in the younger and healthier population has concerning potential ramifications for the general population. If the general population is also utilizing sub-optimal movement strategies, this may contribute to increased risk of injury. In a time when the emphasis is on getting people to be physically active, having a quick screen for overall movement quality could be of value to those professionals who are evaluating and prescribing exercise for the general public.

\section{Inter-rater Reliability}

The two evaluators demonstrated high inter-rater reliability across all four measures of the FSDT. These findings are consistent with the Park and Herman studies, both of which found fair to good measures of inter-rater reliability using Cohen's kappa. ${ }^{1,9}$ However, in this study, the data were treated as ordinal data, while the data from the Park and Herman articles were treated as nominal data. The scores on the FSDT are ordinal, while the interpretation of the data into poor, moderate, or good movement quality is nominal; therefore, since this study examined ordinal data the ICC was used.

\section{Limitations}

The most important limitation of this investigation is the small sample size. Even though number of participants indicated by the $a$ priori power analysis was achieved, the effect size used in the power analysis was an estimate based upon previous work using the FSDT in different study designs. Therefore, follow-up studies to this pilot study would be beneficial to confirm the results of the study. Additionally, the population of the study was relatively homogenous, which limits the validity of the power analysis using data from different populations. Regardless, the pilot nature of this study now provides data for post-hoc analysis for future study development.

The small sample size also limits application of the study's results to different populations. While young, healthy participants were used in the pilot study to assess the FSDT's ability to detect changes after exertion, the test is mainly utilized in patient populations. Therefore, future studies with larger heterogenous samples would allow extrapolation of results to the more general population. Further studies on specific patient population groups would also be beneficial to investigate differences in test response to specific diagnoses.

\section{CONCLUSION}

The FSDT does detect a significant difference in performance quality at five minutes post-exertion, but this difference may or may not be clinically meaningful. Furthermore, a recovery time of ten minutes allows a healthy, young adult to fully recover from a single bout of maximal exertion, returning to their baseline score on the FSDT. However, the large number of "poor" movement quality scores on initial measurement, as well as the lack of any "good" scores, does raise some concerns about the overall fitness levels of physical therapy students in this cohort. This study suggests a more thorough look into the overall fitness level of PT students 
may be warranted given the physical stresses of the occupation they are about to enter, in addition to becoming more aware of applying these concepts to the general public as they are encouraged to become more active themselves.

\section{DECLARATION OF CONFLICT OF INTEREST}

The authors report no conflicts of interest.

\section{APPENDIX 1.}

Instructions for Forward-Step-Down Test

"Stand on the top platform with your hands on your waist. You will be stepping down with your dominant leg (the leg you use to kick a ball) on the step. Place the toes of the dominant leg close to the edge of the top step. The opposite leg should be held in front of you, with your knee straight and your ankle fully dorsiflexed (toes up to the ceiling).

Bend your dominant knee that you are standing on until your non-dominant heel touches the bottom step or until you feel like you cannot go farther. Stand back up. Repeat for a total of 5 times.

You will do the same test now and then again at 1, 5, and 10 minutes after completing the Bruce Protocol. You may stop and withdraw from the study at any time. The investigators have the ability to withdraw you from the study should you not meet the heart rate goal as specified, or if there is any risk above low-risk for you to complete the protocol.

You will have 3 minutes to practice the forward-step-down motion, then we will begin the test."

\section{BIBLIOGRAPHY}

1. Park KM, Cynn HS, Choung SD. Musculoskeletal predictors of movement quality for the forward step-down test in asymptomatic women. J Orthop Sports Phys Ther. 2013;43(7):504-510. [PMID 23756380]

2. Moran KA, Clarke M, Reilly F, Wallace ES, Brabazon D, Marshall B. Does endurance fatigue increase the risk of injury when performing drop jumps? J Strength Cond Res. 2009;23(5):1448-1455. [PMID 19620920]

3. Cortes N, Greska E, Kollock R, Ambegaonkar J, Onate JA. Changes in lower extremity biomechanics due to a shortterm fatigue protocol. J Athl Train. 2013;48(3):306-313. [PMID 23675789]

4. Geiser CF, O'Connor KM, Earl JE. Effects of isolated hip abductor fatigue on frontal plane knee mechanics. Med Sci Sports Exerc. 2010;42(3):535-545. [PMID 19952814]

5. Wesley CA, Aronson PA, Docherty CL. Lower Extremity Landing Biomechanics in Both Sexes After a Functional Exercise Protocol. J Athl Train. 2015;50(9):914-920. [PMID 26285090]

6. Earl JE, Monteiro SK, Snyder KR. Differences in lower extremity kinematics between a bilateral drop-vertical jump and a single-leg step-down. J Orthop Sports Phys Ther. 2007;37(5):245-252. [PMID 17549953]

7. McLean SG, Samorezov JE. Fatigue-induced ACL injury risk stems from a degradation in central control. Med Sci Sports Exerc. 2009;41(8):1661-1672. [PMID 19568192]

8. Loudon JK, Wiesner D, Goist-Foley HL, Asjes C, Loudon KL. Intrarater Reliability of Functional Performance Tests for Subjects With Patellofemoral Pain Syndrome. In: J Athl Train. Vol 37.2002:256-261. [PMID 12937582]

9. Herman G, Nakdimon O, Levinger P, Springer S. Agreement of an Evaluation of the Forward-Step-Down Test by a Broad Cohort of Clinicians With That of an Expert Panel. J Sport Rehabil. 2016;25(3):227-232. [PMID 26655963]

10. Rabin A, Kozol Z. Measures of range of motion and strength among healthy women with differing quality of lower extremity movement during the lateral step-down test. J Orthop Sports Phys Ther. 2010;40(12):792-800. [PMID 20972344]

11. ACSM's Guidelines for Exercise Testing and Prescription. 10 ed. Philadelphia, PA: Wolters Kluwer; 2018.

12. Tanaka H, Monahan KD, Seals DR. Age-predicted maximal heart rate revisited. J Am Coll Cardiol. 2001;37(1):153156. [PMID 11153730]

13. Froelicher VF, Jr., Brammell H, Davis G, Noguera I, Stewart A, Lancaster MC. A comparison of three maximal treadmill exercise protocols. J Appl Physiol. 1974;36(6):720-725. [PMID 4829913]

14. Ros AG, Holm SE, Friden C, Heijne Al. Responsiveness of the one-leg hop test and the square hop test to fatiguing intermittent aerobic work and subsequent recovery. J Strength Cond Res. 2013;27(4):988-994. [PMID 22706574]

15. Baghbani F, Woodhouse LJ, Gaeini AA. Dynamic Postural Control in Female Athletes and Nonathletes After a WholeBody Fatigue Protocol. J Strength Cond Res. 2016;30(7):1942-1947. [PMID 27328275] 
16. Steinberg N, Eliakim A, Zaav A, et al. Postural Balance Following Aerobic Fatigue Tests: A Longitudinal Study Among Young Athletes. J Mot Behav. 2016;48(4):332-340. [PMID 26731202]

17. Bell DR, Padua DA, Clark MA. Muscle strength and flexibility characteristics of people displaying excessive medial knee displacement. Arch Phys Med Rehabil. 2008;89(7):1323-1328. [PMID 18586134]

18. Dierks TA, Manal KT, Hamill J, Davis IS. Proximal and distal influences on hip and knee kinematics in runners with patellofemoral pain during a prolonged run. J Orthop Sports Phys Ther. 2008;38(8):448-456. [PMID 18678957]

19. Kwak SD, Ahmad CS, Gardner TR, et al. Hamstrings and iliotibial band forces affect knee kinematics and contact pattern. J Orthop Res. 2000;18(1):101-108. [PMID 10716285]

20. Powers $\mathrm{CM}$. The influence of abnormal hip mechanics on knee injury: a biomechanical perspective. $J$ Orthop Sports Phys Ther. 2010;40(2):42-51. [PMID 20118526]

21. Racette SB, Inman CL, Clark BR, Royer NK, Steger-May K, Deusinger SS. Exercise and cardiometabolic risk factors in graduate students: a longitudinal, observational study. J Am Coll Health. 2014;62(1):47-56. [PMID 24313696]

22. Chevan J, Haskvitz EM. Do as I do: exercise habits of physical therapists, physical therapist assistants, and student physical therapists. Phys Ther. 2010;90(5):726-734. [PMID 20299411]

23. Peterson DF, Degenhardt BF, Smith CM. Correlation between prior exercise and present health and fitness status of entering medical students. J Am Osteopath Assoc. 2003;103(8):361-366. [PMID 12956248] 